\title{
Capture and photonuclear reaction rates involving charged-particles: Im- pacts of nuclear ingredients and future measurement on ELI-NP
}

\author{
Y. Xu ${ }^{1}{ }^{\star}$, S. Goriely ${ }^{2}$, D.L. Balabanski ${ }^{1}$, S. Chesnevskaya ${ }^{1}$, G.L. Guardo ${ }^{1}$, M. La Cognata ${ }^{3}$, H.Y. Lan ${ }^{4}$, D. Lattuada ${ }^{1}$, \\ W. Luo ${ }^{4}$, and C. Matei ${ }^{1}$ \\ ${ }^{1}$ Extreme Light Infrastructure-Nuclear Physics, RO-077125, Magurele, Ilfov, Romania \\ ${ }^{2}$ Institut d'Astronomie et d'Astrophysique, CP-226, Universite Libre de Bruxelles, 1050, Brussels, Belgium \\ ${ }^{3}$ INFN, Laboratori Nazionali del Sud, Via Santa Sofia 62, 95123, Catania, Italy \\ ${ }^{4}$ School of Nuclear Science and Technology, University of South China, 421001, Hengyang, China
}

\begin{abstract}
The astrophysical p-process is an important way of nucleosynthesis to produce the stable and protonrich nuclei beyond Fe which can not be reached by the s- and r-processes. In the present study, the impact of nuclear ingredients, especially the nuclear potential, level density and strength function, to the astrophysical reaction rates of $(\mathrm{p}, \gamma),(\alpha, \gamma),(\gamma, \mathrm{p})$, and $(\gamma, \alpha)$ reactions are systematically studied. The calculations are performed basad on the modern reaction code TALYS for about 3000 stable and proton-rich nuclei with $12 \leq \mathrm{Z} \leq 110$. In particular, both of the Wood-Saxon potential and the microscopic folding potential are taken into account. It is found that both the capture and photonuclear reaction rates are very sensitive to the nuclear potential, thus the better determination of nuclear potential would be important to reduce the uncertainties of reaction rates. Meanwhile, the Extreme Light Infrastructure-Nuclear Physics (ELI-NP) facility is being developed, which will provide the great opportunity to experimentally study the photonuclear reactions in p-process. Simulations of the experimental setup for the measurements of the photonuclear reactions ${ }^{96} \operatorname{Ru}(\gamma, \mathrm{p})$ and ${ }^{96} \operatorname{Ru}(\gamma, \alpha)$ are performed. It is shown that the experiments of photonuclear reactions in p-process based on ELI-NP are quite promising.
\end{abstract}

\section{Introduction}

In massive star evolution and stellar explosive site, the astrophysical p-process is an important way of nucleosynthesis to produce the stable and proton-rich nuclei beyond Fe which cannot be reached by the s- and r-processes. The common picture is that p-nuclei are synthesized by photodisintegration of pre-existing s- and r-processes nuclei via the capture and photo-disintegration reaction channels. To completely determine the nucleosynthesis of p-process, accurate knowledge of capture and photodisintegration reaction rates for the stable and proton-rich nuclei is essential. However, available experimental information for the reactions involved in p-process is quite limited [1]. For the reactions whose experimental data are not available yet, the state-of-the-art nuclear reaction models and the nuclear structure knowledge deduced by microscopic models should be taken into account for the evaluations of reaction rates.

In the present study, we perform the systematic computations of $(\mathrm{p}, \gamma),(\alpha, \gamma),(\gamma, \mathrm{p})$, and $(\gamma, \alpha)$ reaction rates on about 3000 nuclei based on the TALYS software with various sets of nuclear structure ingredients. Furthermore, simulations of the experimental setup for the measurements of photonuclear reactions of astrophysics interest

\footnotetext{
^e-mail: yi.xu@eli-np.ro
}

in p-process based on the Extreme Light InfrastructureNuclear Physics (ELI-NP) facility are performed, and the preliminarily results are correspondingly presented.

\section{Systematic study on the impacts of nuclear ingredients to capture and photonuclear reaction rates}

The main purpose of the present study is to systematically study how the nuclear structure ingredients, especially the nuclear level density, strength function, and optical model, impact the cross sections and reaction rates of capture and photo-disintegration reactions involving charged particles. Therefore, calculations of (p, $\gamma),(\alpha, \gamma),(\gamma, \mathrm{p})$, and $(\gamma, \alpha)$ reactions on about 3000 stable and proton-rich nuclei are performed with TALYS software[2, 3], by varying the specific nuclear structure ingredients. TALYS provides a complete description of all reaction channels and observables, and takes into account all types of direct, preequilibrium, and compound mechanisms to estimate the total reaction probability as well as the competition between the various open channels.

In particular, 6 sets of nuclear level density (NLD) and 5 sets of strength function (SF) are available in TALYS. The present calculation is performed each time by combining any set of level density models and strength function, 

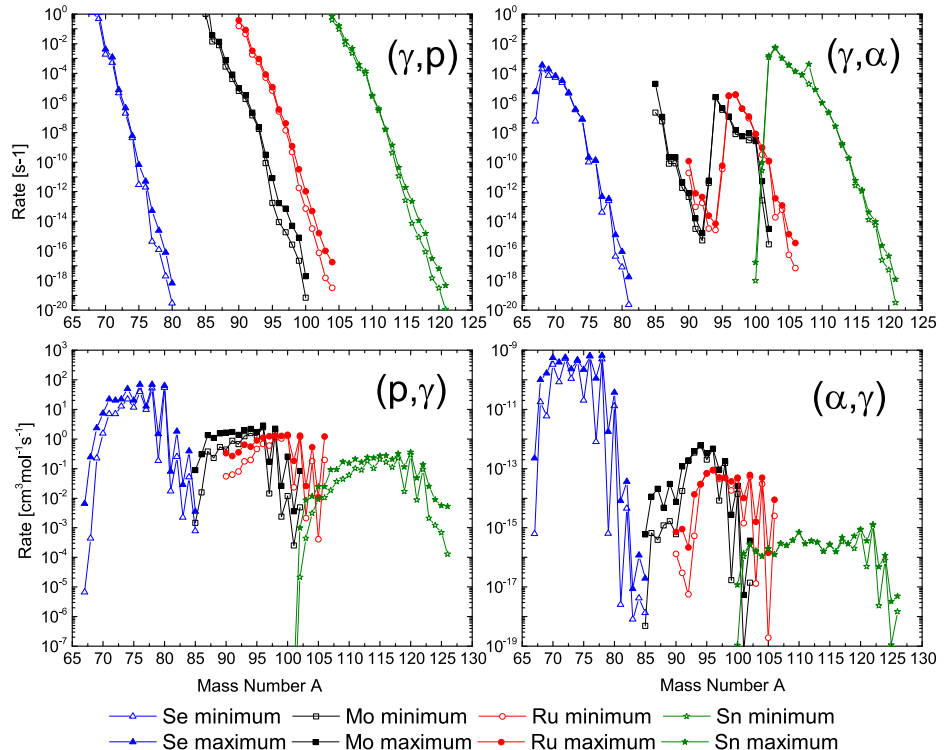

Figure 1. Comparisons of the maximal and minimal values among the reaction rates at $T_{9}=2.5$ calculated by all the available inputs of NLDs and SFs in TALYS for 4 isotopic targets of Se, Mo, Ru and Sn.

thus eventually 30 sets of calculated results are obtained. Among these results, the maximal and minimal values are chosen for comparison. The obtained maximal and minimal reaction rates at $T_{9}=2.5$ for 4 isotopic targets (Se, Mo, $\mathrm{Ru}$ and $\mathrm{Sn}$ ) are shown in figure 1 corresponding to the 4 kinds of reactions. It is found that for the capture reactions, the differences between the maximal and minimal reaction rates are large, which means the capture rate is sensitive to NLD and SF. However, the photonuclear reaction rates are hardly changed by varying the NLD and SF.

Furthermore, calculations of reaction rates for about 3000 stable and proton-rich nuclei using the folding potential (JLM [4] for proton and M3Y [5] for $\alpha$-particle) and the phenomenological Wood-Saxon potential $[6,7]$ are performed. At $T_{9}=2.5$, the ratio of the photonuclear reaction rates calculated by the 2 sets of potentials are shown on the $(\mathrm{N}, \mathrm{Z})$ plane in figure 2 . It is shown that for the reaction involving proton, the ratios are smaller than a factor of 10 for most nuclei. However, the ratios of reaction rates involving $\alpha$-particle are larger than a factor of 100 for most nuclei, which means the rates are very sensitive to the $\alpha$-particle optical model. Therefore, it is proposed to experimentally study the photonuclear reactions based on the ELI-NP facility, especially for the $(\gamma, \alpha)$ channel. The $(\gamma, \alpha)$ reaction rates could be better determined using the improved $\alpha$-particle potential that can be extracted from the measurements of $(\gamma, \alpha)$ reactions.

\section{Simulations of the experimental setup for the measurements of photonuclear reactions based on ELI-NP facility}

A very brilliant gamma beam, produced via Compton backscattering of a laser beam on a relativistic electron beam, will be available at Extreme Light Infrastructure Nuclear Physics (ELI-NP) [8]. The gamma beam is characterized by a narrow bandwidth $(<0.5 \% \mathrm{bdw})$ and tunable energy of up to almost $20 \mathrm{MeV}$. The brilliance of the gamma beam at the peak energy could reach $10^{23} / \mathrm{s}$ $\mathrm{mm}^{2} \mathrm{mrad}^{2}$ with $0.1 \%$ bdw. The research of nuclear astrophysics based on gamma-induced reactions with very low cross sections will largely benefit form the use of the high intensity gamma beams at ELI-NP. Meanwhile, for the detection system of charged particles, silicon detectors represent one of the best solution for their detection because they can guarantee the exceptional energy resolution and they have essentially $100 \%$ efficiency in charged particle detection. In the case of photonuclear reactions of astrophysical relevance, the emitted charged-particles have low energies, ranging from few hundreds $\mathrm{keV}$ to few $\mathrm{MeV}$.

A silicon strip detector array ELISSA, will be realized in a common effort by ELI-NP and INFN Laboratori Nazionali del Sud (INFN-LNS) in Catania, Italy. The prototype of ELISSA was built and tested at INFN-LNS [9]. On this occasion, the simulations of the experimental setup for the measurements of $(\gamma, \alpha)$ and $(\gamma, p)$ reactions on ${ }^{96} \mathrm{Ru}$ target are performed with the help of GEANT4. In the simulation, the configuration of ELISSA is taken 


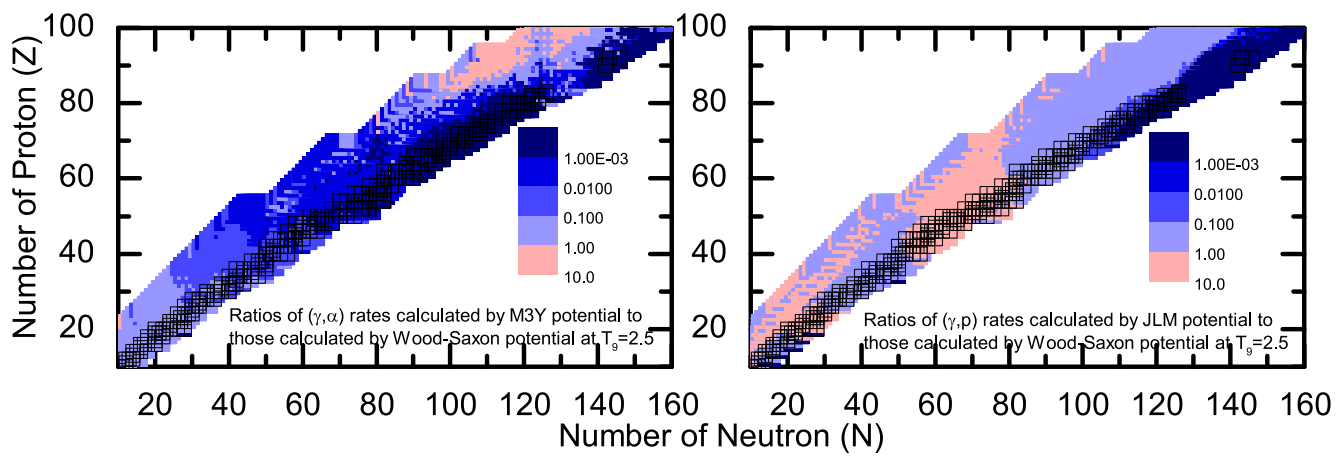

Figure 2. Representation in the $(\mathrm{N}, \mathrm{Z})$ plane of the ratios of $(\gamma, \alpha)$ and $(\gamma, \mathrm{p})$ rates calculated by folding potential to those calculated by Wood-Saxon potential at $\mathrm{T}_{9}=2.5$ for about 3000 stable and proton-rich nuclei.
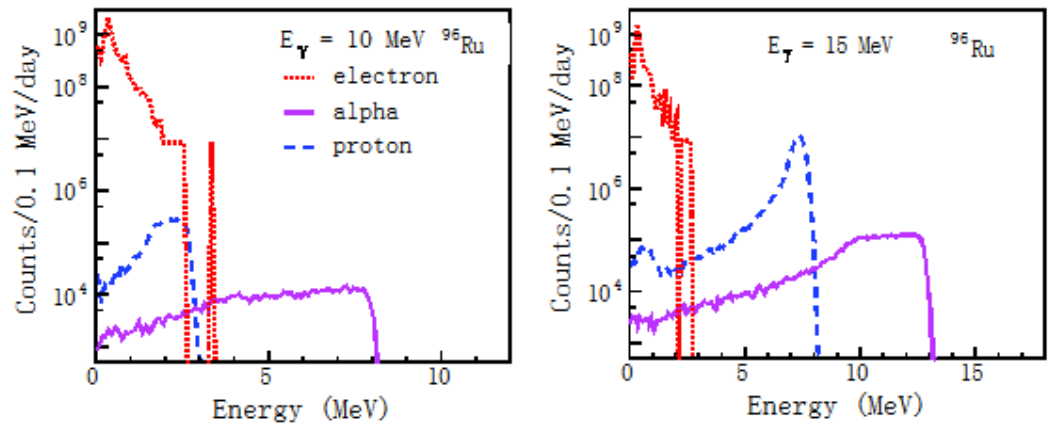

Figure 3. Energy spectrum of proton and alpha particles for ${ }^{96} \mathrm{Ru}(\gamma, \mathrm{p})$ and ${ }^{96} \mathrm{Ru}(\gamma, \alpha)$ reactions obtained by GEANT4 simulations. The incident gamma-ray energies of $10 \mathrm{MeV}$ and $15 \mathrm{MeV}$ are used. The target thickness of Ruthenium is $10 \mu \mathrm{m}$.

into account, and the thickness of ${ }^{96} \mathrm{Ru}$ target is $10 \mu \mathrm{m}$. For the incident gamma-ray energies of $10 \mathrm{MeV}$ and 15 $\mathrm{MeV}$, the energy spectrum of proton and $\alpha$-particle for ${ }^{96} \mathrm{Ru}(\gamma, \mathrm{p})$ and ${ }^{96} \mathrm{Ru}(\gamma, \alpha)$ reactions are shown in figure 3. One can find that $10^{4}$ counts per day can be obtained at $\mathrm{E}_{\gamma}=10 \mathrm{MeV}$ for the experimental yield of $\alpha$-particle, and $10^{5}$ counts per day can be obtained at $\mathrm{E}_{\gamma}=15 \mathrm{MeV}$. We believe that the experiment of the simulated photonuclear reaction is quite promising, and it would be helpful to constraint the $\alpha$-particle potential used for the determination of astrophysical reaction rates.

\section{References}

[1] T. Szücs et al., Nuclear Data Sheets 120, 191 (2014)
[2] A. J. Koning, S. Hilaire, and M. C. Duijvestijn, NRG-report 21297/04.62741/P (2004)

[3] Y. Xu, S. Goriely, A. J. Koning and S. Hilaire, Phys. Rev. C 90, 024604 (2014)

[4] E. Bauge, J. P. Delaroche, and M. Girod, Phys. Rev. C 58, 1118 (1998)

[5] P. Demetriou, C. Grama and S. Goriely, Nucl. Phys. A 707, 253 (2002)

[6] A. J. Koning and J.P. Delaroche, Nucl. Phys. A 713, 231 (2003)

[7] V. Avrigeanu, M. Avrigeanu, and C. Manailescu, Phys. Rev. C 90, 044612 (2014)

[8] C. A. Ur et al., Nucl. Inst. Meth. B 355, 198 (2015)

[9] M. La Cognata et al., JINST 12, C03079 (2017) 